Vol. 7I, N. ${ }^{\circ}$ II4 (noviembre 20I9), 45-59

\title{
¿CONTRIBUYE EL SISTEMA FINANCIERO ECUATORIANO A REDUCIR LA DESIGUALDAD POR INGRESOS? UNA APLICACIÓN DEL ENFOQUE ECONOMÉTRICO ARDL
}

\author{
DOES THE ECUADORIAN FINANCIAL SYSTEM CONTRIBUTE TO REDUCE INCOME \\ INEQUALITY? AN APPLICATION OF THE ARDL ECONOMETRIC APPROACH
}

JEFFERSON SUÁREZ MARTÍNEZ, SAÚL MENDIETA TENESACA

Universidad Central del Ecuador

Recepción manuscrito: 30 de septiembre de 2019

Aceptación versión final: 14 de octubre de 2019

\begin{abstract}
RESUMEN La presente investigación realiza un análisis de la influencia que tiene el desarrollo del sistema financiero ecuatoriano en la desigualdad de ingresos en el periodo 1987-2018. Diversas investigaciones en el mundo han encontrado resultados contradictorios, donde en ciertos países el desarrollo financiero incrementa la desigualdad, mientras que en otros la disminuye, haciendo apremiante determinar qué resultado se ajusta al caso ecuatoriano. De esta manera, utilizando el enfoque econométrico de retraso distribuido autorregresivo (ARDL), se concluye que existen evidencias de que la desigualdad de ingresos se incrementa con el desarrollo del sistema financiero en el periodo estudiado.
\end{abstract}

PALABRAS Clave Índice de Gini, desarrollo financiero, desigualdad, cointegración.

\begin{abstract}
The present investigation carries out an analysis of the influence that the development of the Ecuadorian financial system has on income inequality in the period 1987-2017. Several investigations in the world have found contradictory results, where in certain countries financial development increases inequality, while in others the evolution, making it urgent to determine which result fits the Ecuadorian case. Thus, using the economic approach of distributed self-regressive delay (ARDL), it is concluded that there is evidence of income inequality increases with the development of the financial system in the period studied.
\end{abstract}

KEYWORDS Gini index, financial development, inequality, cointegration.

JEL CODES D53, D63, E6.

\section{INTRODUCCIÓN}

El sistema financiero es un factor de trascendental importancia para el crecimiento de las economías de todo el mundo y de su desarrollo. Su función principal consiste en captar los recursos excedentarios de los agentes económicos y canalizarlos hacia actividades de inversión y consumo. La intermediación financiera, de acuerdo con la teoría económica ortodoxa, permite 
generar mayor producción de bienes y servicios, incrementando la actividad económica y, con ello, el bienestar de todos los integrantes de la sociedad. Sin embargo, de acuerdo con distintas investigaciones, la relación existente entre el desarrollo financiero y desigualdad de ingresos varía de acuerdo con la economía estudiada, ya que en ciertos países o regiones un mayor desarrollo financiero implica mayor desigualdad y en otros casos, sucede todo lo contrario. Dado este escenario, emerge la necesidad de investigar y analizar la relación existente entre el desarrollo financiero y la desigualdad — medida a través del índice de Gini- para el caso ecuatoriano en el periodo 1987-2018.

Estudios relacionados con la desigualdad y el desarrollo del sistema financiero se ha realizado en varios países, es un ejemplo el caso de Pakistán, donde Shahbaz e Islam (2011) concluyeron que «el desarrollo financiero reduce la desigualdad de ingresos, mientras que la inestabilidad financiera lo agrava» (pág. 13). Mientras que, por otro lado, Guerrero (2017), para el caso colombiano, concluyó que el desarrollo financiero incrementó la desigualdad de ingresos entre los años 1994 y 2010. Otros autores como Greenwood y Jovanovic (1990) han predicho la existencia de una relación no lineal entre el desarrollo financiero y la desigualdad, donde la calidad de las instituciones y el nivel de desarrollo financiero condicionan el incremento o la disminución de la desigualdad. Los resultados contradictorios en cada caso analizado hacen apremiante estudiar a profundidad este fenómeno, para así entender su comportamiento.

Por esa razón, el presente trabajo busca analizar cómo el desarrollo del sistema financiero afecta los niveles de desigualdad de ingresos en Ecuador entre los años 1987-2018. La actual investigación se encuentra estructurada en cuatro secciones, en la primera se presenta la teoría económica que estudia el comportamiento de las instituciones financieras y su impacto en la economía, a partir de su visión ortodoxa y su crítica heterodoxa. En la segunda sección se analiza brevemente la historia del sistema financiero en Ecuador, tomando en cuenta el accionar de los grupos financieros en la política pública nacional, sobre todo al finalizar la década de los noventa con la crisis financiera, evidenciando sus características por medio de indicadores históricos y económicos.

En la sección tres se examina rápidamente la incidencia del sistema financiero en la desigualdad por medio de breves argumentos teóricos, mientras se presenta cifras e indicadores macro para el caso ecuatoriano, y así intentar dar respuesta a esta dinámica. En la última sección se plantea la necesidad de demostrar si existe relación entre la desigualdad y el sistema financiero, por medio del enfoque econométrico de cointegración de retrasos distribuidos autorregresivos (ARDL). De esta manera, se examina e interpreta los resultados tomando en cuenta todos los antecedentes mostrados en las secciones anteriores, con lo cual se puede presentar breves reflexiones sobre la influencia del sistema financiero en la desigualdad y emitir algunas sugerencias de política pública.

\section{EL SISTEMA FINANCIERO DESDE EL ENFOQUE ORTODOXO Y LA CRÍTICA HETERODOXA}

La fuente de pensamiento central de la economía y la que más tiene vigencia y cobertura en la actualidad se establece alrededor de dos principios fundamentales: la racionalidad del ser humano y el equilibrio que debe existir en los mercados. La ortodoxia económica, cuyo 
pensamiento alude a los pioneros conservadores como Adam Smith, Alfred Marshall, León Walras, Vilfredo Pareto, entre otros, concibe al sistema financiero como un conjunto de mercados en equilibrio debido a la visión de equilibrio general de León Walras. El mercado de fondos prestables se origina por el equilibrio entre el ahorro y la inversión, dando como resultado la tasa de interés. Este último juega el papel de precio frente a la oferta y demanda de fondos prestables.

La distinción walrasiana entre mercado de fondos prestables y mercados monetarios hace que el dinero tome una posición neutral y sea exógeno. Esto quiere decir, según la visión ortodoxa, la tasa de interés nada tiene que ver con fenómenos monetarios, y la cantidad de dinero no afecta a la producción de bienes y servicios. Según explica De Gregorio (2007):

«[...] en una economía plenamente flexible y competitiva, es decir, donde estamos siempre en pleno empleo, la parte real es determinada en el sector real y la nominal en el sector monetario, y cambios en la cantidad de dinero no tienen efectos reales». (pág. 400)

Dentro de esta concepción, los bancos y demás instituciones financieras dirigen perfectamente todos los recursos que han sido ahorrados por la sociedad en el mercado de fondos prestables hacia la inversión. Esto es consecuencia de la aplicación de la neutralidad del dinero a las finanzas, lo cual implica una estructura financiera que no juega papel alguno en modelos de corte neoclásico, ya que en los mercados financieros perfectos los fondos internos y externos son sustitutos perfectos para las firmas y no hay posibilidad de racionamiento del crédito (Gómez y Reyes, 2002). Debido a esto y al hecho de que las firmas o empresas al interior de una economía competitiva buscan invertir tomando en cuenta su rentabilidad esperada en el futuro, según Gómez y Reyes (2002),

«[...] todas las firmas pueden obtener siempre la cantidad de financiamiento que requieren para poner en marcha sus planes de inversión. Si sus fondos internos no son suficientes, obtienen financiamiento externo al mismo costo y en la cantidad requerida, independientemente de sus posiciones de balance». (pág. 62)

Bajo esta visión donde se dirigen los recursos ahorrados por la sociedad de manera eficiente a la inversión, el papel del Estado sobre el sistema financiero se limita a regulaciones que solamente permitan mantener el equilibrio en los mercados, estableciendo reglas que posibiliten una adecuada competencia. La intervención estatal agresiva, que puede manifestarse por medio de instrumentos de política monetaria y fiscal, son ampliamente rechazados por la ortodoxia, debido a sus efectos en la economía. Estos efectos son considerados como perturbaciones en los ya equilibrados mercados de la «mano invisible», siendo inevitable, bajo estos argumentos, que las consecuencias de las políticas económicas tomen un tono negativo. Precisamente, si el Banco Central intenta implementar una política expansiva incrementando la oferta monetaria, solo se conseguirá incrementar la inflación, todo bajo la premisa ortodoxa donde se considera al dinero como neutro.

Sin embargo, existe una contradicción con la concepción de neutralidad del dinero, sobre todo al hacer referencia a la política monetaria, debido a que se admite la presencia de efectos reales. Friedrich Hayek ya demostró que la neutralidad del dinero no es posible ni en el corto, 
ni en el mediano, ni en el largo plazo, llegando incluso a describirlo como «la ficción del dinero neutral» (Hayek, 1996). Sin embargo, esto no quiere decir que Hayek apruebe la intervención estatal por medio de políticas económicas. Más bien, su rechazo era evidente al considerar que el dinero puede ser objeto de manipulaciones por parte del Estado y, por lo tanto, «la política monetaria es, probablemente, más que el remedio, la causa de las depresiones [...]» (Hayek, 1996, pág. 104).

La neutralidad del dinero ya es un concepto cuestionado por la ortodoxia de la economía, mientras que es rechazado por su contraparte heterodoxa y sirve como insumo para elaborar una de sus tantas críticas. Este conjunto de discrepancias, que nace de la crítica a la rama principal y más predominante del pensamiento económico, muestran al dinero y al sistema financiero como elementos completamente influyentes en los procesos de la economía real. Debido a las diversas crisis financieras que se suscitaron en el siglo xx, era inevitable pensar que las instituciones bancarias podrían causar grandes efectos sobre otras variables de carácter macroeconómico y grave conmoción social. En consecuencia, según el análisis de Gómez y Reyes (2002):

«[...] los trabajos empíricos que se hicieron en esa misma época produjeron resultados inconsistentes con la noción de irrelevancia financiera. Esos resultados, sumados a la creciente fragilidad financiera de las economías a finales del siglo veinte, motivaron al desarrollo de teorías que incluyen explícitamente los factores financieros, en particular el crédito y las estructuras de deuda de las unidades económicas, y que procuran explicar los episodios de crisis financieras como fenómenos económicos». (pág. 62)

Las recurrentes crisis del sistema capitalista motivaron a varios economistas a expresar sus críticas a los clásicos modelos teóricos y empíricos de antaño, ya dados por sentado como abstracciones verdaderas de la realidad. Jhon Keynes, por ejemplo, realizó su obra más famosa, Teoría general del empleo, el interés y el dinero, como una forma de dar explicaciones a la crisis económica estadounidense de 1929. Pese a que Keynes no cuestiona la base central de la teoría walrasiana, es bastante claro al suponer que el dinero no es un ente neutral. En relación con esto, Louis Rochon (2002), en su análisis, menciona que «para ser honestos con Keynes, en una “economía monetaria de producción”, el dinero debe estar ligado a la producción y no debe ser añadido simplemente en cualquier momento o cualquier lugar en el análisis [...]» (pág. 138).

Otros economistas del siglo xx como Nicolás Kaldor abordan la cuestión de si el sistema financiero es neutral. Kaldor (1982) desde el principio duda de la exogeneidad del dinero al considerar que la cantidad monetaria debe estar ligada a su demanda en un sistema de «crédito-dinero», debido a que «el aumento de las transacciones ya sea debido a un aumento en los costos o en el volumen de producción o en ambos, provoca un aumento en la oferta monetaria» (pág. 46). De esa forma, Kaldor concebía a las instituciones financieras como creadoras de dinero fiduciario por medio de sus operaciones de crédito (Kaldor, 1982). Keynes y la escuela poskeynesiana afirman que la tasa de interés no es determinada por la demanda y la oferta de ahorro en el mercado de fondos prestables, sino que se trata de un fenómeno convencional. Esto implica que, según De Juan Asenjo y González (2003), «el interés de equilibrio o de referencia vendría asociado al interés que ha regido en el pasado reciente y se espera que 
prevalezca en el futuro» (pág. 30). Es por esta razón que el Banco Central puede contribuir a fijar la tasa de interés convencional.

Por otro lado, Michal Kaleki es un economista que se aleja de la concepción de equilibrios macroeconómicos y se centra en la presencia de capacidad ociosa no planificada, por lo que entiende al sistema capitalista como una estructura oligopólica a causa de sus raíces marxistas. En consecuencia, él determina tres factores principales para la inversión: los fondos internos de las firmas o empresas, la masa de ganancia y el acervo de capital. Según, explica Levy Orlik (2000) los fondos internos son determinantes del financiamiento interno, y éstos a su vez son determinados por la masa de ganancia de periodos pasados. El capital acumulado y los fondos internos disminuyen el costo del financiamiento y permiten acceder al financiamiento externo. Por lo tanto, el tamaño de la empresa es un factor importante que permite acceder al mercado de capitales y al crédito bancario.

Las empresas más grandes tienen mayores ventajas sobre las pequeñas, por lo que es de esperarse que las primeras, al tener un mayor acceso al mercado de capitales, tengan la capacidad de invertir más con menores costos de financiamiento generando mayores ganancias. En consecuencia, este acceso diferenciado al financiamiento se traduce en restricciones y racionamiento del crédito, que al final establece condiciones de desigualdad. Esta dinámica puede verse agravada aún más por condiciones exógenas que puedan generar un ambiente económico difícil. En efecto, debido al carácter procíclico del grado de racionamiento del crédito, en épocas de auge económico existe menor restricción al crédito, mientras que, durante las recesiones, se producen bruscas restricciones del financiamiento.

\section{SISTEMA FINANCIERO Y GOBIERNO EN LA HISTORIA ECUATORIANA}

La historia financiera ecuatoriana nace bajo la presidencia de García Moreno en 1860, con la creación de la Caja de Amortización de Guayaquil, seguida de la fundación del Banco Continental en 1862 por comerciantes también guayaquileños. La rápida expansión de instituciones financieras en el territorio producto del comercio del cacao, hizo que fuera aceptada una ley de bancos 1967 con preceptos que permitían «aumentar la libertad de abrir establecimientos bancarios, dar a la autoridad el derecho de inspeccionarlos y salvaguardar la soberanía de la emisión y de los bienes de los asociados, ley que facultaba al Estado ser el regulador y supervisor de este naciente sistema financiero (Superintendencia de Bancos, 2016).

El poder acumulado por ciertos bancos inicio la llamada primera guerra bancaria en 1868 entre el Banco Particular, propiedad de Manuel Antonio de Luzarraga, y el Banco del Ecuador, dirigido por Eduardo Arosemena; esta guerra se «caracterizó por difamaciones, negación de aceptación de billetes del banco contrario, e intervención del Ministerio de Hacienda para aceptar o retirar billetes de circulación» (Superintendencia de Bancos, 2016, pág. 9). El poder acumulado por estos y otros bancos llego al nivel donde la política monetaria del Ecuador estaba regida por el Banco Particular, propiedad de Luzarraga, debido a que no existía una ley bancaria en el país.

La ley de bancos y monedas de 1897 que, alineándose con el régimen financiero internacional de patrón oro, dejaba sin permiso de funcionamiento al Banco del Ecuador y al Banco 
Comercial y Agrícola de Guayaquil por no cumplir con la exigencia de mantener al menos el $50 \%$ de su capital respaldado en oro. Esta ley fue objetada por el ministro de Hacienda ${ }^{1}$ el 19 de noviembre de 1898, emitiéndose una nueva Ley de bancos y monedas en 1899, hecha a la medida de los intereses de estas dos instituciones para que puedan funcionar.

Ciertamente, la influencia de las instituciones financieras en la política pública ha estado presente en toda la historia, ya que ciertas veces hasta han actuado como parte del Estado controlando el tipo de cambio, cotizaciones de divisas y fijación de tasas de interés. Historiadores como Óscar E. Reyes aseguran que las decisiones más delicadas del Gobierno ecuatoriano en la década de 1920, como la posesión de senadores, diputados, ministros e incluso candidatos presidenciales, pasaban bajo el escrutinio de Francisco Urbina Jado, gerente del Banco Comercial y Agrícola.

Como respuesta a los altos niveles, desigualdad y desatención por parte de un Estado parcialmente controlado por los grupos financieros y empresariales, se inicia la denominada Revolución Juliana en 1925, con el ascenso al poder de gobiernos progresistas con agendas sociales hasta 1960. «Entre 1948 y 1960 se incorporaron al control de la sBs, la Caja de Ahorro y Crédito Agrícola y nuevos bancos privados que se fundaron, así como las sucursales de bancos extranjeros que se establecieron en el país» (Superintendencia de Bancos, 2016, pág. 17).

A partir de 1981, después del regreso a la democracia, el gobierno de Oswaldo Hurtado (1981-1984) aplicó medidas de corte neoliberal, favoreciendo nuevamente a grupos económicos y financieros a través de la sucretización de la deuda privada. Durante el Gobierno de León Febres Cordero (1984-1988) se realizó una resucretización de las deudas del sector privado con costos perjudiciales para el Estado favoreciendo a las empresas, además amplió el plazo de pago a 7 años, «congeló la tasa de interés en 16\%, ancló el tipo de cambio a 100 sucres por dólar para el sector y, además, extendió el período de gracia para los empresarios de un año y medio a cuatro años y medio» (Paz y Miño Cepeda, 2016, pág. 14).

El poderío acumulado por los grupos económico-financieros se reflejó con la expedición de la Ley de Régimen Monetario en el Art. 78, que fijaba que el 50\% de los miembros de la Junta Monetaria provinieran del sector privado. El poco control estatal establecido en la Ley General de Instituciones del Sistema Financiero expedida por Sixto Durán Ballén en 1994 modificó las actividades tradicionales de la banca en su artículo 23, autorizándoles adquirir acciones y participaciones en instituciones financieras extranjeras y legalizó las empresas offshore. ${ }^{2}$ Otros artículos, como el número 44, les permitían a los bancos ser inversores en actividades ajenas a la banca. Además, y como una de las causas principales de la crisis de 1999, «los arts. 73 y 127 les permitían realizar créditos vinculados a los accionistas, sociedades y directivos» (Paz y Miño Cepeda, 2016, pág. 16).

Las recetas neoliberales presentadas como parte de la «modernización» del sistema financiero, no cumplieron con el objetivo planteado y quebraron una veintena de bancos e instituciones financieras durante el resto de la decana de 1990, lo que redujo a la economía ecuatoriana a una de sus peores crisis, mientras que el Estado destinó millones de dólares al salvataje de los bancos. La crisis fue tan profunda que se tuvo que renunciar a la moneda nacional y adoptar el dólar como la mejor salida a los problemas de devaluación constante del sucre, la desconfianza internacional y la inflación que era muy alta en 1999, lo que minaba la riqueza de los ecuatorianos, su calidad vida y su estado de bienestar. 
El miércoles 11 de marzo, el presidente Mahuad dispuso el congelamiento de los depósitos por un año en cuentas corrientes con más de USD 500 o 2 millones de sucres y las de ahorros con más de USD 5 millones, [...] para proteger la reserva monetaria, que para ese mes contaba con USD 200 millones, para bajar el precio del dólar que bordeaba los 18 mil sucres y para permitir que los clientes cobraran sus depósitos de manera reprogramada como consecuencia del pánico generalizado en todo el sistema financiero naciona. (Superintendencia de Bancos, 2016, pág. 19)

En el año 2000, con la economía dolarizada y parcialmente estabilizada, el Gobierno de Gustavo Noboa (2000-2002) aplica la Ley para la trasformación económica del Ecuador, mejor conocida como ley Trole, que contenía medidas para poder superar la crisis a través de la flexibilización laboral, código orgánico financiero, seguridad social de la policía, contratos petroleros, empresas públicas, organización territorial, zonas especiales de desarrollo, deudas de la banca cerrada, entre otras.

Los gobiernos de corte neoliberal continuaron hasta la llegada a la presidencia en 2007 de Rafael Correa Delgado (2007-2017), el cual instaura un gobierno progresista con una nueva Asamblea Constituyente (2008), creándose así una nueva constitución que prohibió garantizar deudas privadas con recursos públicos. Además, se promulgó la Ley de Creación de la Red de Seguridad Financiera con pilares fundamentales como la supervisión financiera, fondo de liquidez, sistema de seguro de depósitos y esquemas de resolución bancaria. En febrero de 2014 se aprueba la Ley Orgánica para el cierre de la crisis bancaria de 1999, cuyo objetivo fue encaminar el cobro de la cartera vencida y establecer un mecanismo de pago a los acreedores perjudicados por la banca cerrada (Superintendencia de Bancos, 2016, pág. 21).

Con esta ley y otras reformas planteadas en 2017 se pretendía «32.000 emprendimientos del Banco de Fomento (en liquidación), 14.381 operaciones de cartera vencida de BanEcuador y apoyar a 2.228 deudores de buena fe» (ibidem). Además, la Constitución del 2008 y leyes de control financiero facultaron al Estado como el ente regulador y supervisor de todo el sistema financiero, detectando e interviniendo instituciones deterioradas y devolviendo oportunamente los recursos a los depositantes.

Los grupos financieros tacharon a esta ley y al Gobierno de intervencionistas, pues las tasas de interés de todo el sistema se fijan a través de la Junta de Planificación Monetaria y Financiera y se instrumentalizan a través del Banco Central, que es dirigido por el ejecutivo con participación de representantes de grupos económicos y financieros y de la ciudadanía en general.

\section{INCIDENCIA DEL SISTEMA FINANCIERO SOBRE LA DESIGUALDAD}

En un sistema de mercado imperfecto es necesario analizar qué sectores sociales son los más beneficiados por el crecimiento y la acumulación generados a través del desarrollo del sistema financiero. Un ejemplo son las investigaciones realizadas por Galor y Zeira, en la que concluyen que las imperfecciones en el mercado de crédito no permiten, a aquellos individuos con bajos niveles de ingresos, invertir más en actividades de emprendimiento, salud y educación, reproduciendo este esquema a nivel intergeneracional. Diversas investigaciones afirman la existencia de una relación entre desarrollo financiero y desigualdad se dividen en dos ramas; una con relación lineal, es decir, que ha mayor o menor desarrollo financiero existirán mayores o 
menores niveles de desigualdad, mientras que la teoría de relación no lineal propone una relación de U invertida de Kuznets. ${ }^{3}$ Tal es el trabajo realizado por Greenwood y Jovanovic, y concluyen que «el ingreso primero aumenta y después disminuye cuando se alcanzan niveles más altos de desarrollo financiero y cuando una mayor proporción de la población tiene acceso a los crecientes mercados financieros» (1990, pág. 12).

Según datos de la Red de Instituciones Financieras de Desarrollo, hasta el año 2017 existían 8,1 millones personas económicamente activas (PEA), de las cuales 4,2 millones se dedican a la microempresa y, del total de la PEA, solo el $46 \%$ contaba con una cuenta de ahorros, el $23 \%$ solicitó un préstamo y el $32 \%$ ahorró dinero. De aquellos que ahorraron dinero, el 24,8\% correspondió a los más pobres, a diferencia del $36,7 \%$ que representa a los más ricos. Además, «el $25 \%$ utilizaron instrumentos electrónicos, 5,7\% tenían tarjeta de crédito y apenas un o,6\% utilizó su cuenta en una institución financiera para realizar directamente pagos» (Superintendencia de Bancos, Subdirección de Estadisticas y Estudio, 2018, pág. 15).

En Ecuador, en el año 2017 fueron 24 el número de bancos regulados por la Superintendencia, de éstos, 22 son los que cuentan con instrumentos para otorgar microcrédito, cuyas colocaciones supusieron el $6,48 \%$ de la cuota de total para este año. Por lo tanto, cabe destacar que solo el $8 \%$ de los emprendedores del país se financian a través de recursos prestados por el sistema financiero. Además, el 56\% de los hogares ahorran dinero y el $40 \%$ entienden cómo funciona la aplicación de las tasas de interés. Por lo tanto, las cifras nos indican que el país cuenta con un sistema financiero fuerte, pero con acceso deficiente a sus productos, ya sea por la falta conocimiento, sucursales en zonas alejadas o marginadas, pobreza o desempleo, además de factores que hacen difícil la inclusión en el sistema como la falta de recursos y patrimonio de los ciudadanos.

Por otro lado, la evolución de las colocaciones de crédito por parte del sistema financiero en la historia reciente del Ecuador se caracteriza por ser accidentada, con problemas de liquidez y quiebra de instituciones financieras a partir de 1994. La grave crisis de 1998-99 tuvo repercusiones negativas en el sistema financiero hasta el 2003, lo que hizo que el volumen de crédito pase del 22,17\% al 16,64\% del PIB entre 1999 y 2003 (ver Figura 1) y «el pIB medido en dólares cayó en 31\% entre 1998 y 2000, de USD 19.710 millones a USD 13.649 millones» (Acosta, 2006, pág. 194). Dada la grave crisis, el país experimento un acelerado incremento de la pobreza, «entre el año 1995 y el año 2000, el número de pobres se duplicó de 3,9 a 9,1 millones,

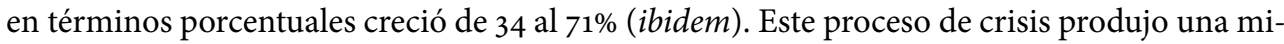
gración de entre 300.000 y 500.000 ecuatorianos, incidiendo profundamente en estructura económica y familiar ecuatoriana.

Los procesos de crisis hicieron que la desigualdad se incrementara rápidamente, en 1990 el índice de Gini se encontraba en o,430 con tendencia a reducirse, pues en 1991 bajó a 0,360 (ver Figura 2). Sin embargo, producto de la creciente inestabilidad financiera y política, además, de los problemas bancarios, salvatajes, quiebra de instituciones financieras y pérdida de la moneda nacional, la desigualdad en el país se incrementó hasta su nivel más alto en el año 2000 con 0,56 puntos. A partir del año 2000 hasta el 2007, la desigualdad se mantuvo por encima de los 0,50 puntos, reflejando el grave problema social en el que se encontraba el país. Dado esto, «[...] el 10\% de los más pobre recibía el 1\% del ingreso, mientras el 10\% más rico 
Figura 1. Evolución del volumen de crédito/PIB

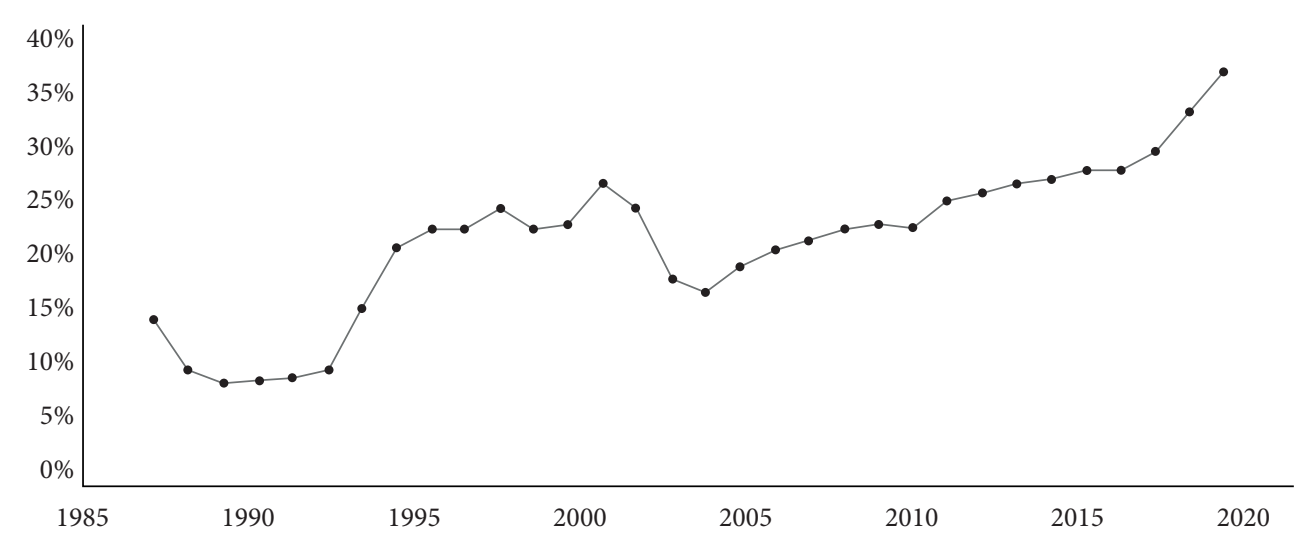

Elaboración: propia.

Figura 2. Evolución del índice de Gini

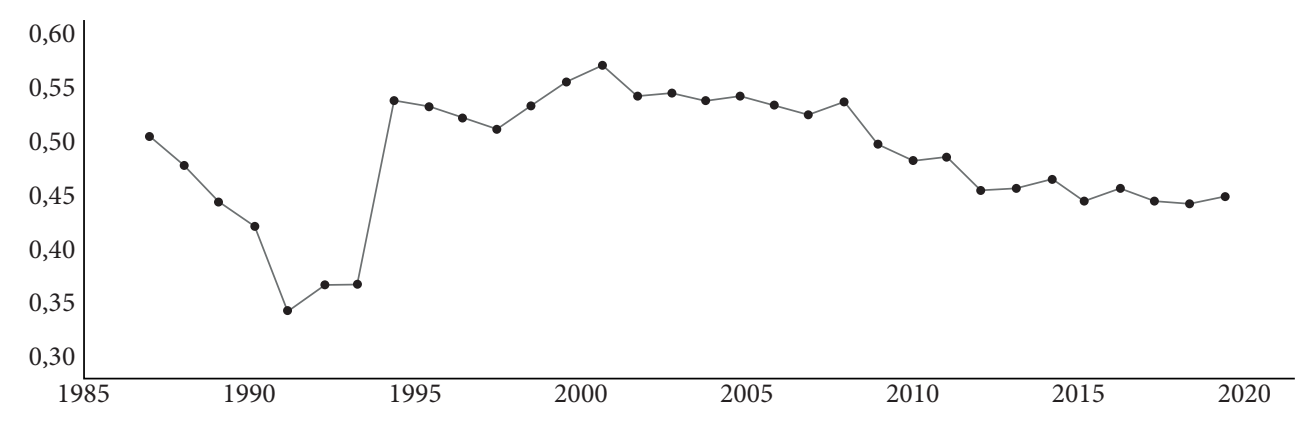

Elaboración: propia.

concentra el 44\% [...]» (Sánchez, 2007, pág. 9). Además, los estudios sobre la pobreza realizados en 2005 demostraban que «[...] el 37\% de los ecuatorianos era pobre y el 10\% era indigente» (Encuesta de Condiciones de Vida, 2005).

Dicho lo cual, el índice de Gini nacional (ver Figura 2), nos muestra cómo la desigualdad se encontraba con tendencia ascendente hasta el año 2007, mientras que para el año 2008, producto de las nuevas políticas sociales implementadas por el Gobierno, el índice de Gini se redujo más de 3 puntos decimales. La tendencia se mantuvo decreciente hasta el año 2012, a partir del cual la desigualdad cambió constantemente, con rápidos períodos de incremento y disminución. La crisis petrolera sufrida en los años 2014 y 2015, la alta dependencia del gobierno de ingresos por la venta de este commodity 4 y su bajo precio, repercutieron negativamente en la economía y en la desigualdad. Además, con un sistema financiero continuamente vigilado y ejerciendo actividades netamente financieras estipuladas en la nueva Ley Bancos 
y la Red de Seguridad Financiera, el volumen de crédito en 2010 aumentó en 31,36\%; en 2011, $18,45 \%$ y en $2012,10,64 \%$.

\section{DESCRIPCIÓN DE LOS DATOS, LA METODOLOGÍA Y EL MODELO}

Para examinar la relación entre desarrollo financiero y desigualdad, a continuación se muestra un modelo empírico representado en la siguiente ecuación:

$$
\text { Gini }=f(\text { FD, FINS, CV) }
$$

El modelo de la ecuación 1 se la puede representar como una función lineal simple. De esta manera se obtiene la ecuación 2:

$$
\text { LnGini }=\beta_{o}+\beta_{1} \ln \mathrm{FD}+\beta_{2} \mathrm{FINS}+\beta_{3} \mathrm{CV}+\mu_{t}
$$

Donde FD representa el desarrollo financiero y esta serie de datos corresponde al crédito interno que es dirigido al sector privado por parte de los bancos y otras instituciones financieras (menos el Banco Central) como porcentaje del PIB. Conviene mencionar que FD es una variable proxy al desarrollo del sistema financiero, mientras que el coeficiente de Gini (GINI) mide la desigualdad en la distribución del ingreso. Debido a que el índice de Gini es sensible sobre todo a los cambios en el ingreso en la parte media de la distribución (Gindling y Trejos, 2004), sección donde se distribuye los ingresos de la clase media que, para el caso de Ecuador, es la que mayor acceso tiene al sistema financiero; este índice se vuelve muy útil para determinar si el sistema bancario afecta los ingresos de las familias.

Por otro lado, la serie FINs hace referencia a la inestabilidad financiera y su cálculo proviene de la metodología de Loayza y Ranciere (2006), que también es usada por Shahbaz e Islam (2011). CV en la ecuación 2 se refiere a un conjunto de variables de control, las cuales son: gasto consumo final de los hogares residentes como proporción del PIB (CONSUMO) proxy del consumo de los hogares, el gasto del Gobierno como proporción del PIB (GASTO) proxy del gasto gubernamental, la apertura comercial como proporción del PIB (COMER) [(importaciones+exportaciones)/PIB] proxy de las relaciones comerciales del país, inflación (INF) y valor agregado bruto del sector manufacturero como proporción del PIB (VABMAN) proxy del desarrollo del sector manufacturero industrial. Todos estos indicadores, que corresponden al periodo 1987-2017, son factores que pueden establecer procesos de acumulación o redistribución del ingreso, así que han sido incluidas en el modelo.

\section{COINTEGRACIÓN DE LOS DATOS}

Existen varios métodos de cointegración, como los propuestos por Engle y Granger y la prueba de cointegración de Johansen $(1991,1995)$, entre otros. Sin embargo, para poder aplicar estos métodos es necesario que todas las variables involucradas estén integradas en el mismo orden, caso contrario, mencionan Shahbaz e Islam (2011) «[...] crea ineficiencia que afecta los poderes predictivos (Kim et al, 2004 y Perron, 1989, 1997)» (pág. 7). Es por esta razón que Pesaran, Shin and Smith (2001) desarrollaron un enfoque de cointegración que se adapta mejor a muestras pequeñas y tolera una combinación de variables cointegradas de orden $I(0)$ y orden $I(1)$; 
características que presentan las series de este trabajo. El enfoque ARDL también permite corregir problemas muy frecuentes dentro de modelos de series temporales dinámicos, tales como la correlación serial residual y el problema de endogeneidad de las variables, según menciona Pesaran, Shin and Smith (1999). Para ello, es necesario modificar apropiadamente las órdenes o retardos de las series.

El modelo ARDL toma la forma descrita en la ecuación 3 conforme a las series que se pretende cointegrar:

$$
\begin{aligned}
& \Delta L G I N I_{t}=c+c_{1} \text { trend }+\beta_{1} L_{\text {LINI }} I_{t-1}+\beta_{2} L F D_{t-1}+\beta_{3} \text { FINS }_{t-1}+\beta_{4} L \text { CONSUMO }_{t-1}+\beta_{5} L_{\text {LASTO }} t-1 \\
& +\beta_{6} L \operatorname{COMER}_{t-1}+\beta_{7} I N F_{t-1}+\beta_{8} L V A B M A N_{t-1}+\sum_{\imath=1}^{p} \alpha_{1 \imath} \Delta L G I N I_{t-l}+\sum_{\imath=0}^{p} \alpha_{2 \iota} \Delta L F D_{t-\imath}
\end{aligned}
$$

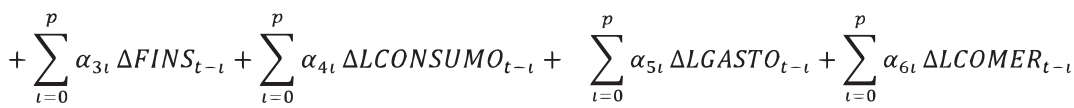

$$
\begin{aligned}
& +\sum_{\iota=0}^{p} \alpha_{7 \iota} \Delta I N F_{t-\iota}+\sum_{\imath=0}^{p} \alpha_{8 \iota} \Delta L V A B M A N_{t-\iota}+\varepsilon_{t}
\end{aligned}
$$

Solamente se hará uso de máximo un rezago en las variables tanto explicativas como explicadas debido al limitado número de datos ( 32 datos) y a la gran cantidad de variables explicativas, 7 en total. Por otro lado, se ha incluido en el modelo un componente determinista: la constante, de modo que se recoja en el modelo la tendencia creciente de las variables FD, GASTO y COMER. Es necesario, mencionar que índice de Gini es una serie construida a partir de varias fuentes, debido al limitado número de datos. Si bien es cierto la fuente principal es el Banco Mundial, debido a que existían muchos vacíos en esa base, sobre todo en el período 19872003 , las fuentes usadas para completar la serie fueron CEPAL y dos investigaciones. Una proveniente de la Facultad Latinoamericana de Ciencias Sociales y otra perteneciente a Andrade Andrade y Vidal Jaramillo (2011).

Para poder comprobar, la condición de cointegración se debe hacer comparaciones con los estadísticos F provistos por Narayan (2005), en donde se establece que si el estadístico F calculado supera el valor crítico del límite superior, existe cointegración o una relación a largo plazo. Si se encuentra entre el límite superior e inferior, no se puede determinar la condición de cointegración; y si se encuentra por debajo del límite inferior, no existe relación de niveles a largo plazo. El número de retardos del modelo ARDL para las variables explicativas se determina por medio de los criterios Akaike (AIC) y Schwarz (SBC) donde, en ambos casos, se escoge el menor valor.

Es necesario que el modelo ARDL supere varias pruebas de diagnóstico ${ }^{5}$ para verificar su calidad, las cuales son: la prueba de normalidad de Jarque-Bera, la prueba de heterocedasticidad ARCH, de correlación serial Breusch-Godfrey y de estabilidad de corto y largo plazo. Para este último, el parámetro de verificación será la suma acumulativa de residuos recursivos (CUSUM) y la suma acumulativa de cuadrados de residuos recursivos (CUsum sq). ${ }^{6}$ 
De acuerdo con la metodología de Pesaran, Shin y Smith (2001), se ha limitado el número de rezagos a una por variable y se ha usado los criterios de optimización AIC y sBC. Al realizar la prueba de cointegración teniendo en cuenta el modelo con la configuración de rezagos escogida, se ha obtenido un estadístico F de 11,66 (ver Tabla 1), con lo cual se ha excedido el valor crítico superior de 4,21 al 5\% de significancia. Esto implica, que existe una condición de cointegración o de relación a largo plazo con un nivel de esperanza del 95\%. El impacto a largo plazo del desarrollo financiero tiene como consecuencia un incremento en la desigualdad, ya que un aumento del $1 \%$ en el desarrollo financiero, incrementa la desigualdad de ingresos en $0,23 \%$ (ver Tabla 1). Estos resultados pueden ser signo de que, para el caso ecuatoriano, no existe un adecuado acceso al financiamiento de los sectores más pobres de la sociedad, y el acceso es limitado para la clase media y las pequeñas y medianas empresas.

Un incremento de la inestabilidad financiera tiende a aumentar gravemente la desigualdad en el caso ecuatoriano. Se estima que un aumento en $1 \%$ de la inestabilidad financiera, puede llegar a incrementar en 4,10\% la desigualdad (ver Tabla 1), sobre todo si esto conlleva una crisis en el sistema. Esto causaría un dramático impacto en la calidad de vida de la sociedad, sobre todo de la clase media y pobre. La ausencia de políticas efectivas que protejan a los cuentaahorristas de corridas bancarias y los procesos de liberalización financiera aplicados sobre todo en la década de los noventa, ha tenido consecuencias negativas en la distribución del ingreso y la riqueza en el largo plazo.

El consumo de las familias está directamente relacionado con una mejora en el ingreso de estas. Por lo tanto, es evidente que un incremento en el consumo de los hogares implique una mejora en la distribución del ingreso. Sin embargo, si el consumo de los hogares no contribuye a reducir la desigualdad, puede implicar que este se ha incrementado en los sectores más pudientes en detrimento de la clase media y pobre. Tal es el caso que, al observar la tabla 1 los resultados muestran que un incremento en $1 \%$ en el consumo de las familias incrementa la desigualdad en $1,80 \%$ en el largo plazo.

Por otro lado, los procesos inflacionarios pueden desencadenar procesos de acumulación, ya que las familias destinan una mayor parte de sus ingresos al consumo, mientras que los sectores más adinerados, al tener altos ingresos, pueden cubrir sin ningún problema su consumo. Para el caso de Ecuador, un incremento del 1\% en la inflación, implica un incremento del índice de Gini del o,49\% en el largo plazo.

El desarrollo del sector manufacturero puede desencadenar procesos redistributivos sobre todo al generar plazas de trabajo en el sector formal que contribuya al incremento en los ingresos de las familias. Al observar la tabla 1 se puede evidenciar que un incremento en $1 \%$ en el valor agregado bruto del sector manufacturero, puede disminuir la desigualdad en el largo plazo en $0,72 \%$.

Debido que las variables GASTO y COMER no son significativas, se puede concluir que no han tenido una incidencia de largo plazo sobre los procesos de desigualdad o redistribución.

\section{MODELO DE CORRECCIÓN DE ERRORES ECM CON COEFICIENTES A CORTO PLAZO}

El comportamiento del desarrollo financiero y sus efectos en la desigualdad de ingresos en el corto plazo se pueden apreciar por medio de un modelo ECM. Su representación empírica se la puede examinar por medio de la ecuación 4: 
Tabla 1: Modelo ARDL específico-resultados a largo plazo

\begin{tabular}{|c|c|c|c|c|}
\hline \multicolumn{5}{|c|}{ Variable dependiente: LGINI (1 retardo) } \\
\hline Retardos & Regresor & Coeficiente & t-statistic & Prob-values \\
\hline- & Constante & $-1,2897$ & 0,4641 & 0,0124 \\
\hline 0 & LFD & 0,2269 & 3,1028 & 0,0061 \\
\hline 1 & FINS & 4,1017 & 2,3707 & 0,0291 \\
\hline 1 & LCONSUMO & 1,7972 & 3,8474 & 0,0012 \\
\hline 0 & LGASTO & $-0,1263$ & $-0,8284$ & 0,4183 \\
\hline 0 & LCOMER & $-0,0614$ & $-0,5047$ & 0,6199 \\
\hline 1 & INF & 0,4869 & 3,6898 & 0,0017 \\
\hline 1 & LVABMAN & $-0,7157$ & $-3,6527$ & 0,0018 \\
\hline \multicolumn{5}{|c|}{$\mathrm{R}^{2}=0,9313$} \\
\hline \multicolumn{5}{|c|}{$\mathrm{Adj}-\mathrm{R}^{2}=0,8856$} \\
\hline \multicolumn{5}{|c|}{ F - Statistics $=20,3465$} \\
\hline \multicolumn{5}{|c|}{ Durbin - Watson $=1,9278$} \\
\hline \multicolumn{5}{|c|}{ Verificación del modelo (Pruebas de diagnóstico)6 } \\
\hline \multicolumn{5}{|c|}{ Serial Correlation LM, F = 0,9368 (0.0339) } \\
\hline \multicolumn{5}{|c|}{ ARCH Test $=0.3796(0.7970)$} \\
\hline \multicolumn{5}{|c|}{ Normality Jarque - Bera Value $=0,5697(0,1254)$} \\
\hline \multicolumn{5}{|c|}{ Heteroscedisticity Test, $\mathrm{F}=0,5961(0,8605)$} \\
\hline \multicolumn{5}{|c|}{ Prueba de cointegración } \\
\hline F - Boun & 11,6587 & & & \\
\hline
\end{tabular}

Nota: ${ }^{*}$ Los valores en paréntesis corresponde a los estadísticos F de cada prueba. Elaboración: propia.

Tabla 2: Modelo ECM-resultados a corto plazo

\begin{tabular}{llll}
\hline & \multicolumn{2}{c}{ Variable Dependiente: $\Delta$ LGINI } & Prob-values \\
\hline Regresor & Coeficiente & t-statistics & 0,0000 \\
\hline Contante & $-0,8333$ & $-11,4263$ & 0,0005 \\
\hline$\Delta$ FINS & 1,4237 & 4,1929 & 0,4369 \\
\hline$\Delta$ LCONSUMO & 0,1416 & 0,7951 & 0,0077 \\
\hline$\Delta$ INF & 0,1539 & 2,9969 & 0,0564 \\
\hline$\Delta$ LVABMAN & 0,2664 & 2,0387 & 0,0000 \\
\hline ECT 1 -1 & $-0,6461$ & $-11,4303$ & \\
\hline $\mathrm{R}^{2}=0,8475$ & & Adj $-\mathrm{R}^{2}=0,8170$ & \\
\hline S.E. Regression $=0,0330$ & & SBC $=-3,5347$ & \\
\hline Sum Squared Resid $=0,0272$ & & F-statistic $=27,7886$ & \\
\hline Log Likelihood Equation $=65,0894$ & Durbin - Watson $=1,9278$ \\
\hline
\end{tabular}

Elaboración: propia. 


$$
\begin{aligned}
& \Delta \mathrm{LGINI}_{t}=c-\left(1-\alpha_{1}\right) \mathrm{ECTT}_{t-1}+\beta_{1} \Delta \mathrm{LFD}_{t}+\beta_{2} \Delta \mathrm{FINS}_{t}+\beta_{3} \Delta \mathrm{LCONSUMO}_{t}+\beta_{4} \Delta \mathrm{LGASTO}_{t} \\
& +\beta_{5} \Delta \mathrm{LCOMER}_{t}+\beta_{6} \Delta \mathrm{INF}_{t}+\beta_{7} \Delta \mathrm{LVABMAN}_{t}
\end{aligned}
$$

Para poder observar y analizar el proceso a corto plazo, es necesario tomar en cuenta el coeficiente del término de corrección de errores $\mathrm{ECT}_{t-1}$, el cual medirá el grado de incumplimiento de la relación a largo plazo de la variable explicada LGINI. Si ECT ${ }_{t-1}$ refleja las respuestas de desequilibrio de LGINI, entonces, se puede establecer algunos puntos en referencia a los resultados empíricos obtenidos: $s_{\text {ECT }}$ se encuentra entre o y -1, la corrección para GINI en el periodo $t$ implica una fracción del error en el periodo $t$-1. Esto quiere decir, que GINI puede converger a su ruta de equilibrio a largo plazo en respuesta al comportamiento de las variables exógenas.

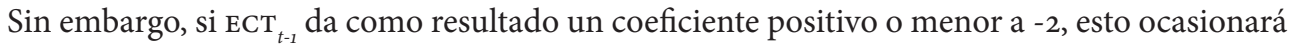
que GINI diverja de su ruta de equilibrio.

Para el caso del presente trabajo, el coeficiente ECT $_{t-1}$ ha tomado un valor negativo entre -1 y o, que es -0,65 (ver Tabla 2). Esto implica que, en condición de desequilibrio, GINI va a corregirse en un $65 \%$ una vez se desplace al próximo periodo, lo cual, al ser significativo al 1\% de tolerancia, nos confirma la condición de cointegración de las variables.

Además, se puede evidenciar que, en el corto plazo, la inestabilidad financiera que puede desencadenar un escenario de crisis puede tener graves efectos en los procesos redistributivos, produciendo condiciones de inequidad en la distribución del ingreso y la riqueza. Los resultados muestran que procesos de inestabilidad financiera pueden generar un incremento en el índice de Gini en un 1,52\% (ver Tabla 2). Por otro lado, cabe mencionar que el desarrollo financiero (FD) y sus efectos en el corto plazo no se han podido determinar por medio de estos resultados. Los procesos inflacionarios tienen repercusiones en la distribución del ingreso en el corto y en el largo plazo. Al observar la tabla 2 se puede observar que un incremento del $1 \%$ en la inflación puede incrementar la desigualdad de ingresos en o,15\% en el corto plazo. Esto puede deberse posiblemente a que los efectos perjudiciales de la inflación, como la disminución del salario real y, a su vez, la disminución del consumo sobre todo de los sectores más vulnerables, se presentan rápidamente en la economía.

Por otro lado, el desigual desarrollo del sector manufacturero, posiblemente debido a los fuertes incentivos por parte del Gobierno a las grandes instituciones financieras y corporaciones más fuertes genera condiciones que incrementan el grado de monopolio en el sector manufacturero industrial en el corto plazo. Aunque en el largo plazo el desarrollo de este sector, contribuye a reducir la desigualdad, en el corto plazo la incrementa. En la tabla 2 se puede observar que un incremento en $1 \%$ en el valor agregado bruto del sector manufacturero, incrementa la desigualdad en $0,27 \%$ al considerar un nivel de significancia del 10\%. Para las variables FD, GASTO y COMER no ha sido posible identificar si estas tienen efecto alguno en la desigualdad en el corto plazo. Mientras tanto, la variable consumo no es significativa en cortos periodos de tiempo.

\section{CONCLUSIONES}

Los resultados obtenidos en la presente investigación muestran que el desarrollo del sistema financiero puede tener una relación directa con un incremento en la desigualdad, medido a través del índice de Gini, en el largo plazo para el caso ecuatoriano. De la misma forma, la 
Figura 3. Prueba de estabilidad CUSUM

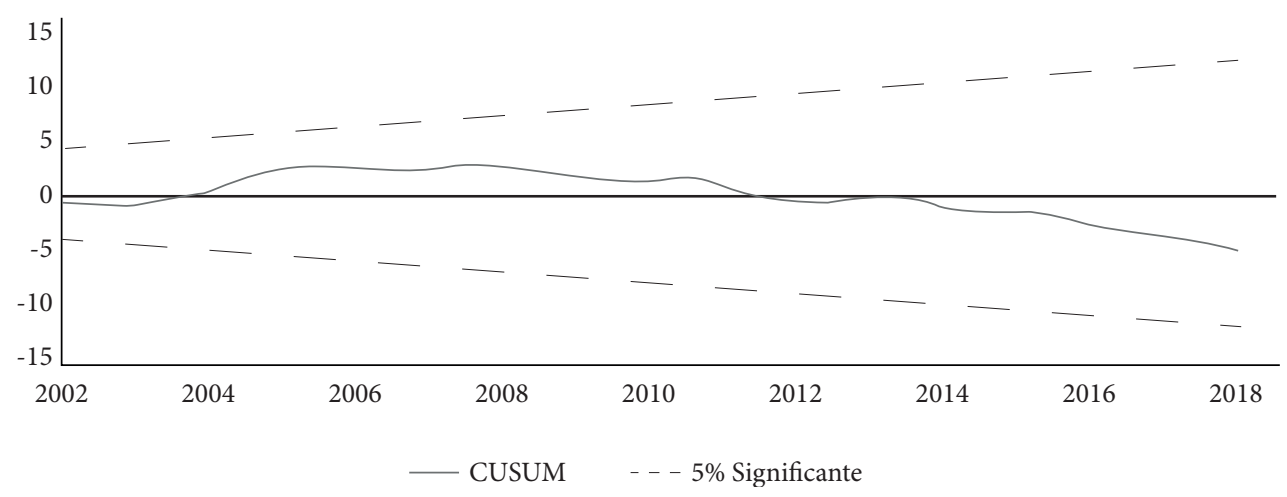

Elaboración: propia.

Figuira 4. Prueba de estabilidad CUSUMsq

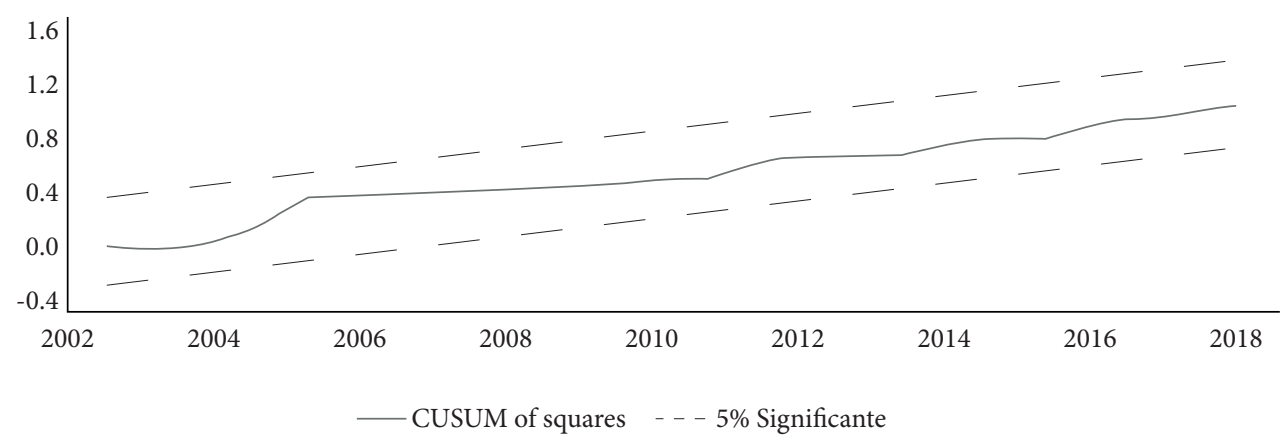

Elaboración: propia.

inestabilidad financiera puede tener graves repercusiones en la redistribución del ingreso, en el corto y en el largo plazo, al afectar directamente a la clase media y baja, sobre todo si se desarrolla y desata corridas y crisis financieras como a finales de los años 9o. Por lo tanto, si bien es cierto es importante el desarrollo de este sector, es necesario aplicar políticas para que este desarrollo sea estable creciente y progresivo.

Por otro lado, el desarrollo del sector manufacturero industrial, a pesar de que en el corto plazo puede llegar a incrementar la desigualdad en el caso de Ecuador, en el largo plazo es necesario llevar a cabo políticas de desarrollo de este sector. Los efectos positivos que se presentan en el tiempo, como el incremento en los puestos de trabajo y la mejora de los salarios y las condiciones laborales, al final se traduce en una mayor redistribución del ingreso. Por lo tanto, es importante implementar políticas que permitan el desarrollo equitativo del sector manufacturero industrial junto con leyes laborales justas, que permitan una adecuada redistribución del ingreso y la riqueza. 
El consumo de las familias ha incrementado los procesos de acumulación en el largo plazo según los resultados presentados. Esto puede relacionarse con la desigualdad estructural que presenta la población en Ecuador, debido a la desigualdad que existe en los ingresos y su distribución en los sectores poblacionales. Los procesos inflacionarios pueden ser una causa de esto, debido a que este afecta directamente a los ingresos de los hogares ocasionando una disminución del consumo en la población más pobre. Es necesario fortalecer la dolarización para que sirva como instrumento de estabilidad y contención de procesos inflacionarios.

El sistema financiero debe ser un actor clave para eliminar la desigualdad y, por ello, cabe hacerse la pregunta de ¿cuál será el impacto real en la economía y en la sociedad ecuatoriana, una mayor inclusión financiera tanto en el corto como en el largo plazo?

\section{NOTAS}

1 Manuel B. Cueva fue el Ministro de Hacienda en dicho periodo.

2 Las empresas offshore se caracterizan por estar constituidas fuera del país de residencia, generalmente en paraísos fiscales con tributaciones cerca del o\%, evadiendo así impuestos en los territorios donde realizan sus actividades económicas.

3 La U invertida de Kuznets hace referencia a las etapas de desarrollo de una nación, afirmando que en un principio los países serían más igualitarios, en una segunda etapa de desarrollo la distribución de la riqueza empeora y se generan focos de concentración de riqueza y poder. En una etapa final, con recursos humanos calificados e instituciones fuertes, se generan procesos de distribución e igualdad de los ciudadanos.

4 El término commodity se utiliza para referirse a bienes genéricos de gran utilidad y poca diferenciación o especialización en el mercado mundial, ejemplo es el petróleo o el trigo.

5 Las pruebas de diagnóstico han sido superadas satisfactoriamente. Sin embargo, debido a la limitada cantidad de datos, que en total suman 32 , estos resultados deben ser interpretados con cautela.

6 Las parcelas para Cusum y cusumsq están entre los límites críticos con un nivel de significancia del $5 \%$. Esto confirma la estabilidad y precisión de los parámetros tanto en el corto como en el largo plazo respecto a su incidencia en la desigualdad de ingresos en el caso de Ecuador (ver Figuras 3 y 4).

\section{REFERENCIAS}

Acosta, A. (2006). Breve historia económica del Ecuador. Quito, Ecuador: Corporación Editoria Nacional.

Andrade Andrade, S. y Vidal Jaramillo, C. (2011). Análisis de la pobreza en el Ecuador a través del índice de Gini y la curva de Lorenz, período 2000-2010. (Tesis de pregrado). Recuperada de Repositorio institucional de la Universidad de Cuenca http://dspace.ucuenca.edu.ec/handle/123456789/1018

De Gregorio, J. (2007). Macroeconomía. Teoría y políticas. Santiago de Chile, Chile: Pearson-Educación.

De Juan Asenjo, Ó. y González Calvet, J. (2003). Un modelo postkeynesiano simple de inestabilidad financiera y ciclos. Revista de Economía Crítica, (2), 25-46.

Gindling, T. y Trejos, J. D. (2004). La desigualdad en Centroamérica durante el decenio de 1990. Revista de la CePAL, (84), 177-198.

Gómez, J. y Reyes, N. (2002). El racionamiento del crédito y las crisis financieras. Revista de Economía Institucional, 4(7), 62-75.

Greenwood, J. y Jovanovic, B. (1990). Financial development, growth, and the distribution of income. The Journal of Political Economy. Recuperado de https://www.jstor.org/stable/2937625? seq=1\#page_scan_tab_contents 
Guerrero, Y. (2017). Colombia: Sistema financiero y pobreza. Revista Ola Financiera, 33-64. Recuperado de http://www.revistas.unam.mx/index.php/RoF/article/view/610o6

Hayek, F. (1996). La desnacionalización del dinero. Barcelona, España: Ediciones Folio.

Instituto Ecuatoriano de Estadística y Censos (INEC). (2005). Encuesta de condiciones de vida. INEC. Recuperado de https://www.ine.es/prensa/np433.pdf

Kaldor, N. (1982). The scourge of monetarism. New York, UsA: Oxford University Press.

Levy Orlik, N. (200o). Kalecki: inversión, inestabilidad financiera y crisis. Comercio Exterior - Banco Nacional de Comercio Exterior s. N. C., 50(12), 1053-1062.

Loayza, N. y Ranciere, R. (2006). Financial development, financial fragility, and growth. Journal of money, credit and banking, (38), 1051-1076.

Narayan, P. (2005). The saving and investment nexus for China: evidence from cointegration tests. Applied Economics, (37), 1979-1990.

Paz y Miño Cepeda. (2016, marzo 1). La crisis bancaria de 1999: el retorno de la «plutocracia»: Historia y Presente. Recuperado de http://www.historiaypresente.com/hyp/wp-content/uploads/2016/11/ crisis-bancaria.pdf

Pesaran, M., Shin, Y. and Richard, J. S. (1999). Bounds testing approaches to the analysis of long run relationships. ESE Discussion Papers, (46), 1-26.

Pesaran, M., Shin, Y. and Smith, R. (2001). Bound testing approaches to the analysis of level relationships. Journal of Applied Econometrics, (16), 289-326.

Rochon, L. (2002). Dinero y dinero endógeno: una aproximación postkeynesiana y de la circulación. Cuestiones Económicas, 18(1:3), 137-168.

Sánchez, J. (2007). Ministerio de Bienestar Social del Ecuador. CEPAL. Recuperado de https://www.cepal. org/sites/default/files/presentations/presentacionjeanettesanchez.pdf

Shahbaz, M. and Islam, F. (2011). Financial development and income inequality in Pakistan: an application of ARDL approach. MPRA Paper n. ${ }^{\circ} 28222,1-20$.

Superintendencia de Bancos. (2016). Memorias. Quito, Ecuador: Superintendencia de Bancos.

Superintendencia de Bancos. (2018, mayo 18). Mecanismos de Inclusión Financiera «IF» en Ecuador. sUPERBANCOS. Recuperado de http://estadisticas.superbancos.gob.ec/portalestadistico/portalestudios/wp-content/uploads/sites/4/downloads/2018/05/Inclusion_financiera_dic_17.pdf 